\title{
Outage Analysis of Multi-node Amplify-and-Forward Relay Networks
}

\author{
Karim G. Seddik, Ahmed K. Sadek, Weifeng Su${ }^{\dagger}$, and K. J. Ray Liu \\ Department of Electrical and Computer Engineering, \\ and Institute for Systems Research \\ University of Maryland, College Park, MD 20742, USA. \\ \{kseddik, aksadek, kjrliu\}@eng.umd.edu \\ ${ }^{\dagger}$ Department of Electrical Engineering, \\ State University of New York (SUNY) at Buffalo, Buffalo, NY 14260, USA. \\ weifeng@eng.buffalo.edu
}

\begin{abstract}
In this paper, we consider the outage probability analysis of multi-node amplify-and-forward relay network with $N$ relay nodes helping the source. We consider a system in which each relay node amplifies the source signal only. We obtain an approximation for the outage probability which is tight at high signal-to-noise ratio (SNR). This tight outage approximation shows that the system can achieve a maximum diversity of order $N+1$. For the case of $N=1$, our approach gives the same result obtained previously by Laneman et. al. for the single relay scenario.
\end{abstract}

\section{INTRODUCTION}

Severe attenuation, due to fading, in wireless networks causes a high degradation in the received signal quality. This makes diversity achieving techniques crucial for the future wireless services. Diversity can have a lot of forms such as spatial diversity, temporal diversity, etc. Spatial diversity has gained much more interest because it can be easily achieved without any delay or rate loss. Achieving the transmit diversity at the mobile users is limited by their space limitations, which makes it difficult to have more than one antenna at the mobile units. In this case, the transmit diversity can be achieved through node cooperation [1], [2], in which the nodes try to form a virtual multiple element transmit antenna. In [3], the classical relay channel model based on additive white Gaussian noise (AWGN) channels was presented. The techniques of cooperative diversity have been introduced, for example, by Sendonaris in the context of CDMA systems [4], [5]. In [2], different protocols were proposed to achieve spatial diversity through node cooperation. Among those protocols was the amplify-and-forward protocol, which has the advantage of simple processing of the received signal at the relay node. It was shown in [2] that the single relay amplify-and-forward protocol will achieve full diversity of order two in terms of outage probability.

In this paper, we consider the outage probability for more general multi-node amplify-and-forward relay net-

\footnotetext{
${ }^{1}$ This work was supported in part by CTA-ARL DAAD 190120011.
}

works with $N$ relay nodes in which each node helps the source by amplifying the the signal it receives from the source only. In [2], the outage probability of the single relay amplify-and-forward network was obtained by considering the high SNR behavior of the outage probability based on the limiting behavior of the cumulative distribution function (CDF) of certain combinations of exponential random variables. We use a simpler approach to find the outage probability of amplify-and-forward relay network with $N$ relay nodes. We also consider the high SNR behavior of the outage probability. The case analyzed in [2] can be considered as a special case of our system with $N=1$ and our result is consistent with that in [2] for that simple case. We will prove that the system in which each relay amplifies only the source signal will achieve full diversity of order $(N+1)$ in this case.

Notations. Lower case and upper case bold letters stands for vectors and matrices, respectively. An exponential random variable $z$ with rate $\lambda$ is a random variable with probability density function (pdf) given by $p_{z}(a)=$ $\lambda e^{-\lambda a}, a \geq 0$.

\section{SySTEM ModeL}

In this section, we introduce the multi-node sourceonly amplify-and-forward system model. The time frame structure of that system is shown in Fig. 1. We consider a cooperative strategy with two phases, In phase 1 , the source transmits its information to the destination, and due to the broadcast nature of the wireless channels the neighbor nodes receive the information. In phase $2, N$ users help the source by amplifying the source signal. In both phases, we assume that the users transmit their information through orthogonal channels (refer to Fig. 1) and perfect synchronization between the cooperating nodes. We focus on one cooperation scenario, however, nodes can interchange their rules as source, relay or destination. 

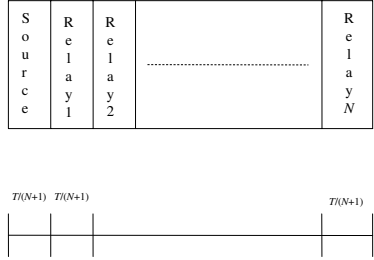

Fig. 1. Time frame structure for the multi-node amplify-and-forward relay network.

In phase 1 , the source broadcasts its information to the destination and $N$ relay nodes. The received signals $y_{s, d}$ and $y_{s, r_{i}}$ at the destination and the $i$ th relay can be written respectively as

$$
\begin{aligned}
y_{s, d} & =\sqrt{P_{s}} h_{s, d} x+\eta_{s, d}, \\
y_{s, r_{i}} & =\sqrt{P_{s}} h_{s, r_{i}} x+\eta_{s, r_{i}}, \quad \forall i \in\{1,2, \ldots, N\},
\end{aligned}
$$

where $P_{s}$ is the transmitted source power, $\eta_{s, d}$ and $\eta_{s, r_{i}}$ denote the additive white Gaussian noise (AWGN) at the destination and the $i$ th relay respectively, and $h_{s, d}$ and $h_{s, r_{i}}$ are the channel coefficients from the source to destination and the $i$ th relay, respectively. Each relay amplifies the received signal from the source and re-transmits to the destination. The received data at the destination in phase 2 due to the $i$ th relay transmission is given by

$$
y_{r_{i}, d}=h_{r_{i}, d} \beta_{i} y_{s, r_{i}}+\eta_{r_{i}, d},
$$

and $\beta_{i}$ satisfies the power constraint, that is

$$
\beta_{i} \leq \sqrt{\frac{P_{i}}{P_{s}\left|h_{s, r_{i}}\right|^{2}+N_{0}}},
$$

where $P_{i}$ is the $i$ th relay power. The channel coefficients $h_{s, d}, \quad\left\{h_{s, r_{i}}\right\}_{i=1}^{N}$ and $\left\{h_{r_{i}, d}\right\}_{i=1}^{N}$ are modeled as zeromean complex Gaussian random variables with variances $\delta_{s, d}^{2}, \quad\left\{\delta_{s, r_{i}}^{2}\right\}_{i=1}^{N}$ and $\left\{\delta_{r_{i}, d}^{2}\right\}_{i=1}^{N}$ respectively. The channel coefficients are assumed to be available at the receiving nodes but not at the source. The noise terms are modeled as zero-mean complex Gaussian random variables with variance $N_{0} / 2$ per dimension.

\section{MUlTi-NODE AMPLIFY-AND-FORWARD RELAY NETWORK MUTUAL INFORMATION}

In this section, we find the mutual information between the source signal and the signals received during the different phases. Let us define an $(N+1) \times 1$ vector $\mathbf{y}=$ $\left[y_{s, d}, y_{r_{1}, d}, \ldots, y_{r_{N}, d}\right]^{T}$. We apply a simple trick to get the mutual information between $x$ and $\mathbf{y}$ by applying maximal ratio combiner (MRC) detector on $\mathbf{y}$ which is different from

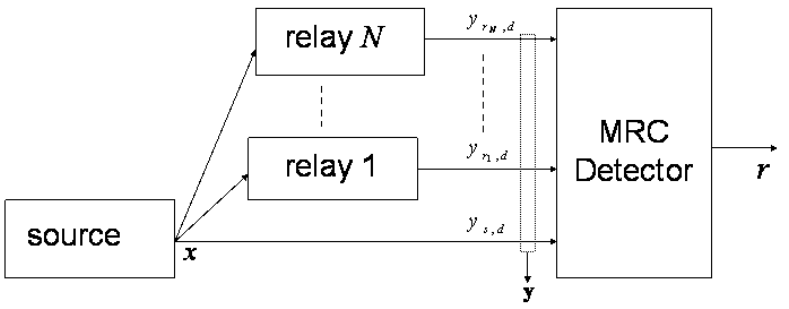

Fig. 2. The multi-node amplify-and-forward relay network system model.

the matrix approach in [2]. The system is shown in Fig. 2. The output of the MRC detector can be given by

$$
r=\alpha_{s} y_{s, d}+\sum_{i=1}^{N} \alpha_{i} y_{r_{i}, d},
$$

where $\alpha_{s}=\sqrt{P_{s}} h_{s, d}^{*} / N_{0}$ and

$$
\alpha_{i}=\frac{\sqrt{P_{s}} \beta_{i} h_{r_{i}, d}^{*} h_{s, r_{i}}^{*}}{\left(\beta_{i}^{2}\left|h_{r_{i}, d}\right|^{2}+1\right) N_{0}} .
$$

We can now write $r$ in terms of $x$ as

$$
\begin{aligned}
r & =\left(\frac{P_{s}\left|h_{s, d}\right|^{2}}{N_{0}}+\sum_{i=1}^{N} \frac{P_{s} \beta_{i}^{2}\left|h_{r_{i}, d}\right|^{2}\left|h_{s, r_{i}}\right|^{2}}{\left(\beta_{i}^{2}\left|h_{r_{i}, d}\right|^{2}+1\right) N_{0}}\right) x+\frac{\sqrt{P_{s}} h_{s, d}^{*}}{N_{0}} \eta_{s, d} \\
& +\sum_{i=1}^{N} \frac{\sqrt{P_{s}} \beta_{i} h_{r_{i}, d}^{*} h_{s, r_{i}}^{*}}{\left(\beta_{i}^{2}\left|h_{r_{i}, d}\right|^{2}+1\right) N_{0}}\left(\eta_{r_{i}, d}+h_{r_{i}, d} \beta_{i} \eta_{s, r_{i}}\right) .
\end{aligned}
$$

The SNR at the MRC detector output is [7]

$$
S N R_{M R C}=\gamma_{s}+\sum_{i=1}^{N} \gamma_{i}
$$

where $\gamma_{s}=P_{s}\left|h_{s, d}\right|^{2} / N_{0}$, and

$$
\gamma_{i}=\frac{P_{s} \beta_{i}^{2}\left|h_{r_{i}, d}\right|^{2}\left|h_{s, r_{i}}\right|^{2}}{\left(\beta_{i}^{2}\left|h_{r_{i}, d}\right|^{2}+1\right) N_{0}} .
$$

It can be easily shown that $r$ is a sufficient statistics for $x$, that is

$$
p_{\mathbf{y} / x, r}(\mathbf{y} / x, r)=p_{\mathbf{y} / r}(\mathbf{y} / r)
$$

where $p_{\mathbf{y} / x, r}(\mathbf{y} / x, r)$ is pdf of $\mathbf{y}$ given $x$ and $r$, and $p_{\mathbf{y} / r}(\mathbf{y} / r)$ is the pdf of $\mathbf{y}$ given $r$.

Since $r$ is a sufficient statistics for $x$, then the mutual information between $x$ and $\mathbf{y}$ equals the mutual information between $x$ and $r$ [11], that is

$$
I(x ; r)=I(x ; \mathbf{y}) .
$$


Then the average mutual information satisfies

$$
\begin{aligned}
I_{A F} & \leq I(x ; r) \\
& \leq \log \left(1+\frac{P_{s}\left|h_{s, d}\right|^{2}}{N_{0}}+\sum_{i=1}^{N} \frac{P_{s} \beta_{i}^{2}\left|h_{r_{i}, d}\right|^{2}\left|h_{s, r_{i}}\right|^{2}}{\left(\beta_{i}^{2}\left|h_{r_{i}, d}\right|^{2}+1\right) N_{0}}\right),
\end{aligned}
$$

with equality for $x$ zero-mean, circularly symmetric complex Gaussian [6].

It is clear that (11) is increasing in terms of $\beta_{i}$ 's, so to maximize the mutual information, the constraint in (4) should be satisfied with equality [2], yielding

$$
\begin{aligned}
I_{A F} & =\log \left(1+\left|h_{s, d}\right|^{2} S N R_{s, d}\right. \\
& \left.+\sum_{i=1}^{N} f\left(\left|h_{s, r_{i}}\right|^{2} S N R_{s, r_{i}},\left|h_{r_{i}, d}\right|^{2} S N R_{r_{i}, d}\right)\right),
\end{aligned}
$$

where $S N R_{s, d}=S N R_{s, r_{i}}=P_{s} / N_{0} \quad \forall i \in[1, N]$ and $S N R_{r_{i}, d}=P_{i} / N_{0} \quad \forall i \in[1, N]$ and

$$
f(v, u)=\frac{u v}{u+v+1} .
$$

Let $P_{s}=P_{i}=P, \forall i \in[1, N]$ and define $S N R=P / N_{0}$, then we can write

$$
\begin{aligned}
I_{A F} & =\log \left(1+\left|h_{s, d}\right|^{2} S N R\right. \\
& \left.+\sum_{i=1}^{N} f\left(\left|h_{s, r_{i}}\right|^{2} S N R,\left|h_{r_{i}, d}\right|^{2} S N R\right)\right) .
\end{aligned}
$$

\section{Outage Analysis of the Multi-node AMPLIFY-AND-FORWARD RELAY NETWORK}

In this section, we perform the outage probability analysis of the multi-node amplify-and-forward relay network with $N$ relay nodes helping the source by amplifying the source signal only. The outage probability for spectral efficiency $R$ is defined as

$$
P_{A F}^{\text {out }}(S N R, R)=\operatorname{Pr}\left[\frac{1}{N+1} I_{A F}<R\right],
$$

and the $1 /(N+1)$ factor comes from the time frame structure in Fig. 1. Equation (14) can be rewritten as

$$
\begin{aligned}
& P_{A F}^{\text {out }}(S N R, R)=\operatorname{Pr}\left[\left(\left|h_{s, d}\right|^{2} S N R+\sum_{i=1}^{N}\right.\right. \\
& \left.\left.f\left(\left|h_{s, r_{i}}\right|^{2} S N R,\left|h_{r_{i}, d}\right|^{2} S N R\right)\right)<\left(2^{(N+1) R}-1\right)\right] .
\end{aligned}
$$

At high SNR we can neglect the 1 term in the denominator of the $f(.,$.$) function [8]; So we can write the outage$ probability as,

$$
\begin{aligned}
& P_{A F}^{\text {out }}(S N R, R) \simeq \operatorname{Pr}\left[\left(\left|h_{s, d}\right|^{2} S N R+\right.\right. \\
& \left.\left.\sum_{i=1}^{N} \frac{\left(\left|h_{s, r_{i}}\right|^{2} S N R\right)\left(\left|h_{r_{i}, d}\right|^{2} S N R\right)}{\left|h_{s, r_{i}}\right|^{2} S N R+\left|h_{r_{i}, d}\right|^{2} S N R}\right)<\left(2^{(N+1) R}-1\right)\right] .
\end{aligned}
$$

Define $w_{1}=\left|h_{s, d}\right|^{2} S N R$ and $w_{i+1}=$ $\frac{\left(\left|h_{s, r_{i}}\right|^{2} S N R\right)\left(\left|h_{r_{i}, d}\right| S N R\right)}{\left|h_{s, r_{i}}\right|^{2} S N R+\left|h_{r_{i}, d}\right| S N R}, \quad \forall i \in[1, N]$. The outage probability is now given as

$$
P_{A F}^{\text {out }}(S N R, R) \simeq \operatorname{Pr}\left[\sum_{j=1}^{N+1} w_{j}<\left(2^{(N+1) R}-1\right)\right] .
$$

The random variable $w_{1}$ is an exponential r.v. with rate $\lambda_{1}=\frac{N_{0}}{P_{s} \delta_{s, d}^{2}}$. To calculate the outage probability in (17), it is quite challenging to follow the approach in [2]. We consider an alternative approach based on approximating the harmonic mean of two exponential random variables to be exponential random variable.

The $w_{j}$ 's for $j \in[2, N+1]$ are the harmonic mean of two exponential random variables. The CDF for $w_{j}, j=$ $2, \ldots, N+1$ is given by [8]

$$
\begin{aligned}
P_{w_{j}}(w) & =\operatorname{Pr}\left\{w_{j}<w\right\} \\
& =1-2 w \sqrt{\zeta_{j 1} \zeta_{j 2}} e^{-w\left(\zeta_{j 1}+\zeta_{j 2}\right)} K_{1}\left(2 w \sqrt{\zeta_{j 1} \zeta_{j 2}}\right),
\end{aligned}
$$

where $\zeta_{j 1}=\frac{N_{0}}{P_{s} \delta_{s, r_{j-1}}^{2}}, \zeta_{j 2}=\frac{N_{0}}{P_{j-1} \delta_{s, r_{j-1}}^{2}}$ and $K_{1}($.$) is$ the first order modified Bessel function of the second kind defined in [13]. The function $K_{1}($.$) can be approximated$ as $K_{1}(x) \simeq \frac{1}{x}$ for small $x$ [13] from which we can approximate the CDF of $w_{j}$ at high SNR as

$$
P_{w_{j}}(w)=\operatorname{Pr}\left\{w_{j}<w\right\} \simeq 1-e^{-w\left(\zeta_{j 1}+\zeta_{j 2}\right)},
$$

which is the $\mathrm{CDF}$ of an exponential random variable of rate $\lambda_{j}=\frac{N_{0}}{P_{s} \delta_{s, r_{j-1}}^{2}}+\frac{N_{0}}{P_{j-1} \delta_{r_{j-1}, d}^{2}}$. We will check the validity of this approximation in the simulation section by comparing the exact moment generating function (MGF) expressions for both random variables at high SNR.

Define the random variable $W=\sum_{j=1}^{N+1} w_{j}$, the CDF of $W$, assuming the $\lambda_{i}$ 's to be distinct, can be obtained to be

$$
\operatorname{Pr}[W \leq w]=\sum_{k=1}^{N+1}\left(\prod_{m=1, m \neq k}^{N+1} \frac{\lambda_{m}}{\lambda_{m}-\lambda_{k}}\right)\left(1-e^{-\lambda_{k} w}\right) .
$$

The outage probability can be expressed in terms of the CDF of $W$ as

$$
P_{A F}^{\text {out }}(S N R, R) \simeq \operatorname{Pr}\left[W \leq\left(2^{(N+1) R}-1\right)\right] .
$$

The CDf of $W$ can now be written as

$$
\begin{aligned}
& \operatorname{Pr}[W \leq w]=\sum_{k=1}^{N+1} \\
& \left(\prod_{m=1, m \neq k}^{N+1} \frac{\lambda_{m}}{\lambda_{m}-\lambda_{k}}\right)\left(\sum_{n=1}^{N+1}(-1)^{n+1} \lambda_{k}^{n} \frac{w^{n}}{n !}\right)+\text { H.O.T. }
\end{aligned}
$$


where H.O.T. stands for the higher order terms. Rearranging the terms in (20) we get

$$
\begin{aligned}
& \operatorname{Pr}[W \leq w]=\sum_{n=1}^{N+1} \\
& \left(\sum_{k=1}^{N+1}\left(\prod_{m=1, m \neq k}^{N+1} \frac{\lambda_{m}}{\lambda_{m}-\lambda_{k}}\right) \lambda_{k}^{n}\right)(-1)^{n+1} \frac{w^{n}}{n !}+\text { H.O.T. }
\end{aligned}
$$

To prove that the system has diversity order on $(N+1)$ we need to have the coefficients of $w^{n}$ to be zero for $n \in$ $[1, N]$. This requirement can be reformulated in a matrix form as

$$
\underbrace{\left[\begin{array}{ccc}
\lambda_{1} & \ldots & \lambda_{N+1} \\
\lambda_{1}^{2} & \ldots & \lambda_{N+1}^{2} \\
\vdots & \vdots & \vdots \\
\lambda_{1}^{N+1} & \ldots & \lambda_{N+1}^{N+1}
\end{array}\right]}_{\mathbf{V}} \underbrace{\left[\begin{array}{c}
\prod_{m=2}^{N+1} \frac{\lambda_{m}}{\lambda_{m}-\lambda_{1}} \\
\prod_{m=1, m \neq 2}^{N+1} \frac{\lambda_{m}}{\lambda_{m}-\lambda_{2}} \\
\vdots \\
\prod_{m=1}^{N} \frac{\lambda_{m}}{\lambda_{m}-\lambda_{N+1}}
\end{array}\right]}_{\mathbf{q}}=\left[\begin{array}{c}
0 \\
0 \\
\vdots \\
c_{1}
\end{array}\right]
$$

To prove (22), consider the following system of equations

$$
\mathbf{V a}=\underbrace{[0,0, \ldots, 1]^{T}}_{\mathbf{c}}
$$

and prove that $\mathbf{q}=c_{1} \mathbf{a}$ for some constant $c_{1}$. Noting that the columns of the $\mathbf{V}$ matrix are scaled versions of the columns of a Vandermonde matrix, i.e., it is a nonsingular matrix, the solution for the system of equations in (23) can be found as

$$
a=\mathbf{V}^{-1} \mathbf{c}=\frac{1}{\operatorname{det}(\mathbf{V})} \operatorname{adj}(\mathbf{V}) \mathbf{c}
$$

The determinant of a Vandermonde matrix is given by [12]

$$
\operatorname{det}\left[\begin{array}{cccc}
1 & 1 & \ldots & 1 \\
\lambda_{1} & \lambda_{2} & \ldots & \lambda_{N+1} \\
\vdots & \vdots & \vdots & \vdots \\
\lambda_{1}^{N} & \lambda_{2}^{N} & \ldots & \lambda_{N+1}^{N}
\end{array}\right]=\prod_{k=1}^{N+1} \prod_{m>k}^{N+1}\left(\lambda_{m}-\lambda_{k}\right)
$$

from which we can express the determinant of the $\mathbf{V}$ matrix as

$$
\operatorname{det}(\mathbf{V})=\left(\prod_{j=1}^{N+1} \lambda_{j}\right) \prod_{k=1}^{N+1} \prod_{m>k}^{N+1}\left(\lambda_{m}-\lambda_{k}\right)
$$

Due to the structure of the c vector, we are only interested in the last column of the $\operatorname{adj}(\mathbf{V})$ matrix. The $i$ th element of the a vector can be given as

$$
\begin{aligned}
& a_{i}= \\
& \frac{(-1)^{N+i-1}\left(\prod_{j=1, j \neq i}^{N+1} \lambda_{j}\right) \prod_{k=1, k \neq i}^{N+1} \prod_{m>k, m \neq i}^{N+1}\left(\lambda_{m}-\lambda_{k}\right)}{\left(\prod_{j=1}^{N+1} \lambda_{j}\right) \prod_{k=1}^{N+1} \prod_{m>k}^{N+1}\left(\lambda_{m}-\lambda_{k}\right)} \\
& =\frac{(-1)^{N}}{\lambda_{i}} \prod_{j=1, j \neq i}^{N+1} \frac{1}{\left(\lambda_{j}-\lambda_{i}\right)} .
\end{aligned}
$$

From (27), it is clear that $\mathbf{q}=c_{1} \mathbf{a}$ where

$$
c_{1}=(-1)^{N} \prod_{i=1}^{N+1} \lambda_{i}
$$

The outage probability can now be expressed as

$$
\begin{aligned}
& P_{A F}^{\text {out }}(S N R, R) \simeq \operatorname{Pr}\left[W<\left(2^{(N+1) R}-1\right)\right] \\
& =\frac{1}{(N+1) !}\left(\prod_{i=1}^{N+1} \lambda_{i}\right)\left(2^{(N+1) R}-1\right)^{N+1}+\text { H.O.T. }
\end{aligned}
$$

Substituting for the $\lambda_{i}$ 's, we have

$$
\begin{aligned}
P_{A F}^{\text {out }}(S N R, R) \sim & \frac{1}{(N+1) !} \cdot \frac{1}{\delta_{s, d}^{2}} . \\
& \prod_{i=1}^{N} \frac{\delta_{s, r_{i}}^{2}+\delta_{r_{i}, d}^{2}}{\delta_{s, r_{i}}^{2} \delta_{r_{i}, d}^{2}}\left(\frac{2^{(N+1) R}-1}{S N R}\right)^{N+1} .
\end{aligned}
$$

For the special case of single relay node $(N=1)$ we have

$$
P_{A F}^{o u t}(S N R, R) \sim \frac{1}{2} \cdot \frac{1}{\delta_{s, d}^{2}} \cdot \frac{\delta_{s, r_{1}}^{2}+\delta_{r_{1}, d}^{2}}{\delta_{s, r_{1}}^{2} \delta_{r_{1}, d}^{2}}\left(\frac{2^{2 R}-1}{S N R}\right)^{2},
$$

which is consistent with the result obtained in [2] for single relay amplify-and-forward network.

From the expression in (30), it is clear that the multi-node amplify-and-forward network with $N$ relay nodes helping the source by amplifying the source signal only will achieve full diversity of order $N+1$.

\section{SIMULATION RESULTS}

In this section, we present simulations to prove the theoretical analysis presented in the previous sections. First, we compare the exact expression of the MGF of the harmonic mean of two exponential random variables in [9] with the MGF of an exponential random variable. In Fig. 3, all the channel variances are taken to be 1 and the SNR is taken to be $S N R=10,20$ and $30 \mathrm{~dB}$. From Fig. 3 it is clear that approximating the harmonic mean of two exponential random variables to be an exponential random variable is tight at high SNR. 


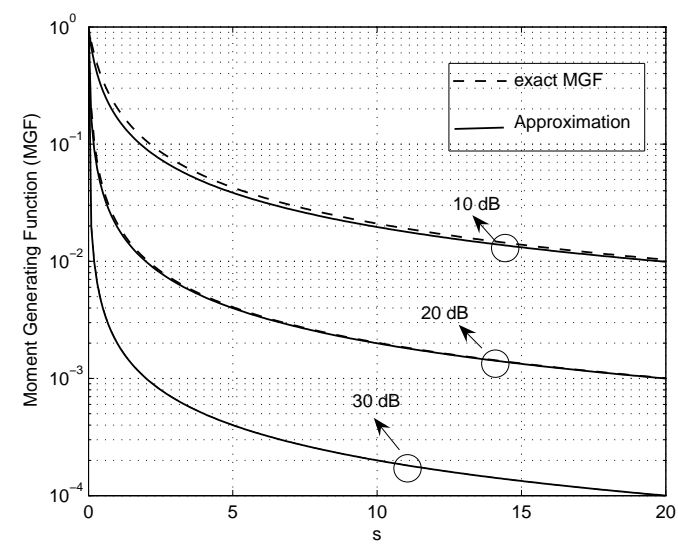

Fig. 3. Exact MGF of the harmonic mean of two exponential random variables versus the MGF of an exponential random variable.

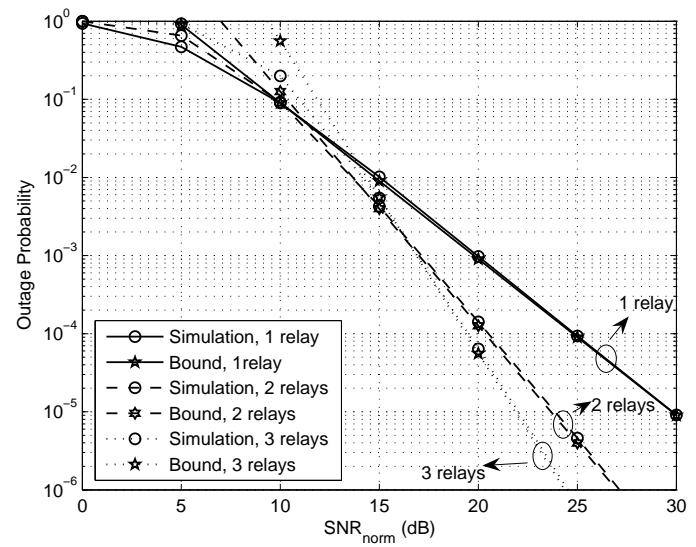

Fig. 4. Outage probability for one and two nodes amplify-and-forward relay network.

Fig. 4 shows the outage probability for one and two relay nodes helping the source versus $S N R_{\text {norm }}$ defined as [10]

$$
S N R_{\text {norm }}=\frac{S N R}{2^{R}-1},
$$

which is the SNR normalized by the minimum SNR required to achieve spectral efficiency $R$ for complex additive white Gaussian noise (AWGN) channel [10]. In the simulations, we used $R=1$ (small $\mathrm{R}$ regime). For the single relay case all the channel variances are taken to be 1, i.e., $\delta_{s, d}^{2}=1, \quad \delta_{s, r_{1}}^{2}=1$ and $\delta_{r_{1}, d}^{2}=1$. For the case of two relay nodes all the channel variances are taken to be 1 except for the channel between the source and the second relay for which the channel variance is taken to be $\delta_{s, r_{2}}^{2}=10$, which means that the second relay is close to the source. From Fig. 4 it is clear that the obtained outage probability bound is tight at high SNR and that the multinode amplify-and-forward system, in which each relay only amplifies the source signal, will achieve the full diversity offered by the system which is $(N+1)$ in the case of $N$ relay nodes helping the source.

\section{CONCLUSION}

In this paper, we have performed the outage analysis for the multi-node amplify-and-forward relay networks with $N$ relay nodes helping the source. We considered a system in which each relay node amplifies only the source signal. We found an outage probability bound which is tight at high SNR. From the obtained bound, it is clear that the system can achieve a maximum diversity order $N+1$, without the need o use other relays forwarded copies of the source signal. We have used a simple approach by approximating the harmonic mean of two exponential random variables to be the minimum of the two random variables (that it is approximating the harmonic mean to be an exponential random variable) to prove that the multi-node amplify-andforward system, in which each relay only amplifies the source signal, can achieve full diversity. The result obtained by Laneman $e t$. el. for the simple case of single relay node helping the source can be considered as a special case of our approach and can be obtained by substituting $N=1$ in the outage probability bound we derived.

\section{REFERENCES}

[1] J. N. Laneman and G. W. Wornell, "Distributed space-time coded protocols for exploiting cooperative diversity in wireless networks", IEEE Trans. Information Theory, vol. 49, pp. 2415-2525, Oct. 2003.

[2] J. N. Laneman, D. N. C. Tse and G. W. Wornell, "Cooperative diversity in wireless networks: efficient protocols and outage behavior", IEEE Trans. Information Theory, vol. 50, no. 12, pp. 3062-3080, Dec. 2004.

[3] T. M. Cover and A. A. El Gamal, "Capacity theorems for the relay channel”, IEEE Trans. Inform. Theory, 25(5):572-584, Sept. 1979.

[4] A. Sendonaris, E. Erkip, and B. Aazhang, "User cooperation diversityPart I: system description," IEEE Trans. Comm., vol. 51, pp.19271938, Nov. 2003.

[5] A. Sendonaris, E. Erkip, and B. Aazhang,, "User cooperation diversityPart II: implementation aspects and performance analysis," IEEE Trans. Comm.,vol. 51, pp.1939-1948, Nov. 2003.

[6] E. Telatar, "Capacity of multi-antenna Gaussian channels", European Transactions on Telecommunications, Vol. 10, No. 6, pp. 585-595, Nov./Dec. 1999.

[7] D. G. Brennan, "Linear diversity combining techniques", Proceedings of the IEEE, vol. 91, no. 2, pp. 331-356, Feb. 2003.

[8] M. O. Hasna and M. S. Alouini , "Performance analysis of two-hop relayed transmission over Rayleigh fading channels ", in Proc. IEEE Vehicular Technology Conf. (VTC), vol. 4, pp. 1992-1996, Sept. 2002.

[9] W. Su, A. K. Sadek and K. J. R. Liu, "Cooperative Communications in wireless networks: performance analysis and optimum power allocation", submitted to IEEE Trans. Inform. Theory, in revision.

[10] L. Zheng and D. N. C. Tse, "Diversity and multiplexing: A fundamental tradeoff in multiple antenna channels", IEEE Transactions on Information Theory, vol. 49, pp. 1073-1096, May 2003.

[11] T. Cover and J. Thomas, Elements of information theory, Wiley, 1991.

[12] R. A. Horn and C. R. Johnson, Matrix analysis, Cambridge Univ. Press, 1985.

[13] M. Abramowitz and I. A. Stegun, Handbook of Mathematical Functions with Formulas, Graphs, and Mathematical Tables. New York, NY: Dover Publications, ninth ed., 1970. 